\title{
ESTUDO NUMÉRICO DE UM LEITO FLUIDIZADO BORBULHANTE UTILIZANDO UM CÓDIGO COMBINADO CFD-DEM
}

\author{
F. C. COLMAN ${ }^{1 *}$, M. J. ALBA ${ }^{2,3 * *}$, P. R. PARAÍSO ${ }^{2}$, A. M. S. $\operatorname{COSTA}^{1}$, L. M. M. JORGE${ }^{2}$ \\ ${ }^{1}$ Universidade Estadual de Maringá, Departamento de Engenharia Mecânica \\ ${ }^{2}$ Universidade Estadual de Maringá, Departamento de Engenharia Química \\ ${ }^{3}$ Universidade Tecnológica Federal do Paraná, Câmpus Apucarana, Departamento de Física \\ e-mails: *fccolman@uem.br; **marceloalba@utfpr.edu.br
}

\begin{abstract}
RESUMO
Leitos fluidizados são usados em indústrias nas operações de separação, mistura, secagem e combustão. Este trabalho analisa o comportamento de um leito fluidizado com o código livre MFIX-DEM, desenvolvido pelo Laboratório Nacional de Tecnologias Energéticas (NETL-USA). Nesse código, as fases sólida e gasosa são tratadas numa abordagem euleriana-lagrangeana. Para a fase gasosa, foram resolvidas as equações da continuidade e da conservação de quantidade de movimento. Para as partículas, ou a fase sólida, foi utilizado o Método dos Elementos Discretos (DEM), que descrevem os fenômenos de contato e colisão entre as partículas com o conceito de mola-amortecedor-atrito, por meio de uma abordagem numérica explícita. Uma malha bidimensional, com injeção de gás na região inferior do leito, foi utilizada. Grãos de soja foram o material granular analisado. A velocidade do gás foi variada para determinar a influência na perda de carga do leito e a curva da velocidade de mínima fluidização. Também foram analisados leitos preenchidos com diferentes alturas iniciais $(0,08 \mathrm{~m}, 0,115 \mathrm{~m}$ e $0,150 \mathrm{~m})$. Os resultados obtidos por diferentes correlações de arrasto foram comparados com curvas de fluidização experimentais.
\end{abstract}

\section{INTRODUÇÃO}

Leitos fluidizados são extensivamente utilizados em diversas áreas industriais como as de agroindústria, químicas e farmacêuticas (KUNII e LEVENSPIEL, 1991). Em geral, os leitos fluidizados possibilitam melhorias consideráveis nas trocas térmicas e de massa, o que representa redução de espaço e custos nas indústrias. Atualmente, muitos estudos tratam também da análise de sistemas gássólido, com um amplo crescimento no uso de recursos computacionais para previsão dos comportamentos de trocas de calor, massa e quantidade de energia.

Especificamente para o caso dos grãos de soja, os leitos fluidizados e também os leitos de jorro são utilizados para transferir calor para os grãos através da injeção de ar aquecido. Em contato com os grãos, o ar eleva a temperatura do ambiente proporcionando a evaporação da umidade contida nos grãos. Este processo é fundamental para permitir o armazenamento dos grãos por longos períodos, sem que eles sofram deterioração por microrganismos. Soponronnarit et al. (2001), afirma que os grãos de soja são colhidos com alto teor de humidade $(25-33 \%$ em base seca) e que o armazenamento seguro requer a diminuição rápida da umidade para preservar a qualidade.

Os leitos de jorros são preferencialmente utilizados na fluidização dos grãos de soja, devido à classificação das partículas no grupo D de Geldart. Apesar disto, leitos fluidizados podem ser utilizados em situações especiais, como aqueles onde o equipamento já existe 
para secagem de outros tipos de grãos ou partículas. Além disto, a secagem por meio de leitos fluidizados é reconhecida como uma tecnologia de secagem rápida, devido à ampla área de contato do produto com o ar, quando comparado aos leitos estáticos, sendo isso consequência da fluidização, da velocidade do ar e das temperaturas elevadas (SOPONRONNARIT et al., 2001). Os grãos de soja também permitem boa aproximação do modelo numérico aqui utilizado para a fase sólida, o Método dos Elementos Discretos (DEM) (CUNDALL e STRACK 1979; TSUJI et al., 1993). A soja tem boa circularidade e esfericidade e uma baixa variabilidade no tamanho dos grãos, o que a torna propícia para o uso comparativo entre métodos experimentais e métodos numéricos.

A aplicação de CFD (Dinâmica dos fluidos computacional) nas indústrias de processo tem levado a reduções nos custos dos produtos e nas atividades de desenvolvimento e otimização, reduzindo a necessidade de experimentação física, aumentando a confiabilidade em melhorias de projeto, e aumento das conversões e rendimentos. Os benefícios econômicos trazidos pela utilização de CFD têm sido substanciais, embora raramente sejam relatadas análises econômicas detalhadas (GIDASPOW, 1994).

Há duas abordagens sendo utilizadas para simulações de escoamentos multifásicos: Euler-Lagrange e Euler-Euler. A primeira, que envolve o balanço de forças que atuam sobre a partícula, requer esforços computacionais consideráveis. A abordagem Euler-Euler considera a fase dispersa como contínua e baseia-se nas equações de NavierStokes aplicadas em cada fase (WEN e YU, 1966; ANDERSON e JACKSON, 1967; SYAMLAL e O'BRIEN, 1989; GIDASPOW et al., 1992; TAGHIPOUR et al., 2005; DUARTE et al., 2009; ASEGEHEGN et al., 2011).

Um aspecto importante e que possui considerável influência sobre a acuidade dos resultados da simulação é o método de obtenção de dados. O método de obtenção e análise de resultados de simulação, bem como, a definição das propriedades do leito, tais como propriedades de bolha e expansão do leito, variam amplamente entre as pesquisas e pouca atenção foi dada por muitos deles (ASEGEHEGN et al., 2011). O método de aquisição de dados influi nos resultados e no processo de validação de forma similar à utilização de diferentes modelos de equações constituintes. Por exemplo, HULME et al. (2005) mostraram que diferentes valores de entrada para a fração volumétrica de sólidos para definir as condições de fronteira da bolha, levou a diâmetros de bolha diferentes da média. Na literatura também é possível encontrar diferentes formas para definição da razão de expansão do leito (LOFSTRAND et al., 1995; GELDART, 2004; TAGHIPOUR et al., 2005). Estas diferentes formas de aquisição e definição de parâmetros de entrada podem ocasionar diferentes desvios entre os resultados experimentais e numéricos e podem não representar o problema em questão de forma consistente.

Outro parâmetro de grande influência sobre os resultados de simulação é o período médio para análise das propriedades do leito fluidizado de acordo com a sua média temporal. PATIL et al. (2005), por exemplo, demonstraram que para diferentes períodos de simulação ocorreram diferentes diâmetros médios de bolha. $\mathrm{O}$ intervalo de período de amostragem depende de diferentes parâmetros, como geometria do leito e velocidade superficial e torna-se, portanto, difícil prover um valor geral para todas as geometrias e condições de operação. Sabe-se que longos períodos de simulação determinam bons resultados, por outro lado, demandam de grande capacidade computacional.

Um dos parâmetros mais importantes que caracterizam um leito fluidizado é a velocidade de mínima fluidização $\left(u_{m f}\right)$. Sua determinação é de extrema importância, apesar 
de poucos processos operarem na condição de mínima fluidização. Segundo Law e Mujumbar (2006), leitos fluidizados operam, normalmente, com velocidades de gás equivalentes entre 2 e $4 u_{m f}$.

Neste estudo nós revisitaremos o fenômeno de fluidização de um leito gasoso, formado por partículas de grãos de soja. Serão abordadas três configurações de altura de leito para cinco correlações de coeficientes de arrasto. O código aberto de dinâmica dos fluidos computacional empre gado foi o MFIXDEM (Multiphase Flow with Interphase eXchange coupled with Discrete Element Method). Os resultados serão comparados com os dados experimentais de Soponronnarit et al. (2001).

\section{DESCRIÇÃO DO MODELO}

O MFIX é um código computacional aberto desenvolvido pela NETL (Laboratório Nacional de Tecnologias Energéticas) com o propósito geral de descrever a fluidodinâmica, transferência de calor e reações químicas em sistemas sólido-fluido. O MFIX resolve equações diferenciais parciais de conservação de massa, momento, espécies e energia para múltiplas fases e promove resultados transientes em três dimensões para pressão, velocidade, temperatura e frações mássicas de espécies químicas.

A Figura 1 representa o domínio para as simulações numéricas. Os pontos para medição da pressão estão localizados em $\mathrm{y}=0,0 \mathrm{~m} / \mathrm{s}$ (entrada de ar) e y $=1,0 \mathrm{~m} / \mathrm{s}$ (saída de pressão). As simulações foram baseadas nos experimentos de Soponronnarit et al. (2001) em um leito gasoso formado por grãos de soja, operado para três diferentes alturas do leito: $0,080 \mathrm{~m}, 0,115 \mathrm{~m}$ e $0,15 \mathrm{~m}$. Uma malha em plano cartesiano bidimensional foi empregada com um arranjo de 20 x 100 células. Testes em malhas com maior número de células foram realizados e atestaram a independência do tamanho da malha para o caso em estudo.
Foram consideradas 13 velocidades para o gás sendo que este distribuído uniformemente pela seção transversal inferior do leito. A partícula possuía um diâmetro de $0.007 \mathrm{~m}$ e densidade de $1200 \mathrm{~kg} / \mathrm{m}^{3}$.

Figura 1 - Malha do leito fluidizado

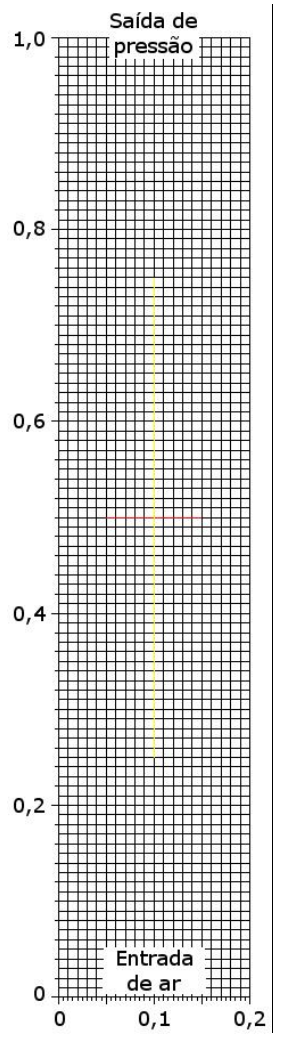

Fonte: Os autores (2015)

Do ponto de vista de interação entre partículas, existem três tipos de escoamento classificados em: (1) Escoamento livre de colisões; (2) Escoamento dominado por colisões; (3) Escoamento dominado por contatos. O terceiro tipo ainda se subdivide em aqueles cujo efeito do fluido é negligenciado e os que este efeito é considerado (TSUJI, 2007). Seguindo esta classificação, os leitos fluidizados estão inseridos no caso (3) em que o efeito do fluido é considerado.

Uma segunda classificação proposta por Tsuji (2007), baseia os métodos de análise numérica a partir da escala de tamanho, onde as partículas e o fluido são classificados nas escalas micro, meso e macro. As fronteiras 
entre estas escalas dependem dos objetivos da pesquisa e dos pontos de vista do pesquisador, de forma que, em geral, os macro modelos são mais empíricos e o micro modelos mais teóricos. Para o estudo aqui proposto, será utilizado o método $\mathrm{E}$, onde modelos de micropartícula e de meso-fluido são considerados. Este método é conhecido pelo rastreamento das partículas (particle tracking) da abordagem lagrangeana, e para o fluido conhecido como método das médias locais (local averaging) da aboradagem euleriana.

\subsection{Método dos elementos discretos (DEM)}

O DEM, também conhecido por método dos elementos distintos, inicialmente proposto por Cundall e Strack (1979), é um método numérico capaz de descrever o comportamento de conjuntos de discos e esferas. Baseado no uso de um esquema numérico explícito, o método monitora a interação das partículas contato por contato e o movimento das partículas é modelado partícula por partícula. Este método é também conhecido como abordagem por esfera macia (do inglês softsphere).

Cundall e Strack (1979) compararam os resultados do método dos elementos distintos com resultados experimentais. Os experimentos foram realizados utilizando-se de materiais opticamente sensíveis descritos como materiais fotoelásticos, que permitem uma determinação precisa das forças de contato, assim como dos deslocamentos e rotações individuais dos discos, porém a um elevado custo temporal. Concluíram que, apesar da comparação realizada ser qualitativa e, portanto, subjetiva, houve uma correspondência suficientemente boa entre os resultados para concluir que o DEM é um instrumento válido para a investigação do comportamento de conjuntos granulares. Ainda hoje, softwares livres e comerciais recorrem ao método para descrever dinamicamente o movimento de partículas em colisão, o que inevitavelmente ocorre no escoamento de partículas sendo fluidizadas. Estes contatos e choques são descritos numericamente como um trio atrito-molaamortecedor, onde é possível estabelecer como as partículas interagem entre si.

Devido ao conceito numérico existente, foi necessário estabelecer quais são as propriedades dos materiais com relação ao coeficiente de atrito, a rigidez da mola e coeficiente de amortecimento. Estas e outras informações, como a densidade dos grãos de soja e o coeficiente de restituição tiveram seus valores aproximados pelos dados apresentados por Boac et al. (2009). Na Tabela 1 são apresentadas as propriedades do material granular e do gás utilizadas no MFIX-DEM.

As simulações foram feitas com velocidades que variaram de $0,45 \mathrm{~m} / \mathrm{s}$ até $3,5 \mathrm{~m} / \mathrm{s}$ com 13 incrementos não uniformes. Para cada velocidade, o tempo de simulação foi de $3 \mathrm{~s}$.

As partículas foram dispostas no leito de forma aleatória. Para tanto, as partículas foram posicionadas com espaços em intervalos não regulares, o que levou a uma elevação da altura do leito. Quando a simulação se iniciou, sob ação da força da gravidade, as partículas caíram de suas posições iniciais, preenchendo a altura do leito especificada no trabalho Soponronnarit et al. (2001).

\subsection{Equações governantes: fase gasosa}

No MFIX-DEM, as equações governantes da fase gasosa para conservação de massa e de quantidade de movimento são similares àquelas tradicionais da fase gasosa de CFD, mas com termos adicionais devido ao arrasto da fase sólida. A fase sólida é modelada utilizando as partículas discretas. As equações governantes da continuidade e da quantidade de movimento, implementadas no MFIX (Syamlal et al., 1993), para a fase gasosa são:

$$
\frac{\partial}{\partial t}\left(\varepsilon_{g} \rho_{g}\right)+\nabla\left(\varepsilon_{g} \rho_{g} \overrightarrow{v_{g}}\right)=0
$$


Tabela 1 - Propriedades físicas, condições experimentais e condições iniciais e de contorno usadas nas simulações CFD-DEM.

\begin{tabular}{|c|c|c|c|}
\hline \multicolumn{2}{|c|}{ Propriedades } & Valores da literature & Valores utilizados \\
\hline \multirow{2}{*}{ Coeficiente de atrito estático, $\mu_{\mathrm{s}}$} & Partícula-partícula & 0,$267 ; 0,55^{(1)}$ & 0,45 \\
\hline & Partícula-parede ${ }^{(4)}$ & $0,30^{(1)}$ & 0,30 \\
\hline \multirow{2}{*}{ Coeficiente de restituição, $e$} & Partícula-partícula & 0,$5 ; 0,7^{(1)}$ & 0,6 \\
\hline & Partícula-parede ${ }^{(4)}$ & 0,$6 ; 0,7^{(1)}$ & 0,6 \\
\hline \multicolumn{2}{|l|}{ Diâmetro da partícula $\left(\mathrm{x} 10^{-3} \mathrm{~m}\right)$} & & $7^{(3)}$ \\
\hline \multicolumn{2}{|c|}{ Comprimento da partícula $\left(\times 10^{-3} \mathrm{~m}\right)$} & $7,0-8,2^{(1)}$ & -- \\
\hline \multicolumn{2}{|c|}{ Largura da partícula $\left(\times 10^{-3} \mathrm{~m}\right)$} & $6,1-6,7^{(1)}$ & -- \\
\hline \multicolumn{2}{|l|}{ Espessura da partícula $\left(\times 10^{-3} \mathrm{~m}\right)$} & $5,5-5,9^{(1)}$ & -- \\
\hline \multicolumn{2}{|c|}{ Densidade do sólido, $\left(\mathrm{kg} / \mathrm{m}^{3}\right)$} & $1130-1325,2^{(1)}$ & 1200 \\
\hline \multicolumn{2}{|c|}{ Altura das partículas no leito, $\left(\mathrm{x} 10^{-2} \mathrm{~m}\right)$} & 8,$0 ; 11,5 ; 15,0^{(2)}$ & 8,$0 ; 11,5 ; 15,0$ \\
\hline \multicolumn{2}{|c|}{ Altura do leito $\left(\times 10^{-2} \mathrm{~m}\right)$} & $100^{(2)}$ & 100 \\
\hline \multicolumn{2}{|l|}{ Largura do leito (x10-2 m) } & $20^{(2)}$ & 20 \\
\hline \multicolumn{2}{|c|}{ Densidade do gás $(\operatorname{ar}), \rho_{\mathrm{g}}\left(\mathrm{kg} / \mathrm{m}^{3}\right)$} & & 1.205 \\
\hline \multicolumn{2}{|c|}{ Variação das velocidades de entrada do ar, $v_{\mathrm{g}}(\mathrm{m} / \mathrm{s})$} & $0,45-3,5^{(2)}$ & $0,45-3,5$ \\
\hline
\end{tabular}

${ }^{(1)}$ Boac et al. (2009) $\quad{ }^{(3)}$ Considerada esférica

${ }^{(2)}$ Soponronnarit et al. (2001) $\quad{ }^{(4)}$ Acrílico

Fonte: Os autores (2015)

$$
\frac{D}{D t}\left(\varepsilon_{g} \rho_{g} \vec{v}_{g}\right)=\nabla \cdot \overline{\bar{S}}_{g}+\varepsilon_{g} \rho_{g} \vec{g}-\sum_{m=1}^{M} I_{g m}
$$

Em que, $\varepsilon_{g}$ é a fração volumétrica da fase gasosa, $\rho_{g}$ a densidade da fase gasosa, $\vec{v}_{g}$ o vetor velocidade para a fase gasosa, $I_{g m}$ é o termo de quantidade de momento transferida entre a fase gasosa e a $\mathrm{m}^{\text {th }}$ fase sólida, e $\overline{\bar{S}}_{g}$ é o tensor tensão da fase gasosa dado por:

$$
\overline{\bar{S}}_{g}=-P_{g} \overline{\bar{I}}+\overline{\bar{\tau}}_{g}
$$

onde $P_{g}$ é a pressão para a fase gasosa. O tensor tensão no regime viscoso, $\overline{\bar{\tau}}_{g}$, é assumido como newtoniano e assume a seguinte forma:

$$
\overline{\bar{\tau}}_{g}=2 \mu_{g} \overline{\bar{D}}_{g}+\lambda_{g} \operatorname{tr}\left(\overline{\bar{D}}_{g}\right) \overline{\bar{I}}
$$

e o tensor $\overline{\bar{I}}$ pode ser identificado como o tensor taxa de deformação para a fase fluida $\overline{\bar{D}}_{g}$, dada por:

$$
\overline{\bar{D}}_{g}=\frac{1}{2}\left[\nabla \vec{v}_{g}+\left(\nabla \vec{v}_{g}\right)^{T}\right]
$$

\subsection{Equações governantes: fase sólida}

Na aproximação pelo DEM, a fase sólida $m^{\text {th }}$ é representada por partículas esféricas $N_{m}$ com cada partícula possuindo um diâmetro $D_{m}$ e densidade $\rho_{s m}$. As fases sólidas são diferenciadas baseadas em suas densidades e diâmetros. Para o somatório de todas as fases sólidas $m$, o número total de partículas é igual a:

$$
N=\sum_{m=1}^{N} N_{m}
$$

Estas N partículas são representadas em uma abordagem lagrangeana com referência no tempo por:

$$
X^{(i)}(t), V^{(i)}(t), \omega^{(i)}(t), D^{(i)}, \rho^{(i)} \quad i=1 \ldots . . N
$$

onde $X^{(i)}(t)$ denota a posição da $i^{\text {th }}$ partícula, $V^{(i)}(t)$ e $w^{(i)}(t)$ denotam as velocidades linear e angular, $D^{(i)}$ equivale ao diâmetro, e $\rho^{(i)}$ representa a densidade. 
A posição e as velocidades angular e linear da $i^{\text {th }}$ partícula devem ser encontradas a partir da Lei de Newton:

$$
\begin{aligned}
& \frac{d X^{(i)}(t)}{d t}=V^{(i)}(t) \\
& m \frac{d V^{(i)}(t)}{d t}=F_{T}^{(i)} \\
& F_{T}^{(i)}=m^{i} g+F_{d}^{(i \in k, m)}(t)+F_{c}^{(i)}(t) \\
& I^{i} \frac{d w^{(i)}(t)}{d t}=T^{i}
\end{aligned}
$$

em que $g$ é a aceleração da gravidade, $F_{d}^{(i \in k, m)}$ é a força de arrasto total sobre a $i^{\text {th }}$ partícula presente na $k^{\text {th }}$ célula e pertencente a $m^{\text {th }}$ fase sólida, $F_{c}^{(i)}$ é o conjunto de forças de contato devido ao contato com outras partículas, $T^{i}$ é a soma de todos os torques agindo sobre a $\mathrm{i}^{\text {th }}$ partícula, e $F_{T}^{(i)}$ é a soma total de todas as forças atuando sobre a $i^{\text {th }}$ partícula.

Para o cálculo da força de arrasto, utilizase do seguinte modelo:

$$
\begin{aligned}
F_{d}^{(i \in k, m)}=-\nabla \mathrm{P}_{\mathrm{g}}\left(\mathrm{x}_{\mathrm{k}}\right) & \\
& +\frac{\beta_{\mathrm{m}}^{(\mathrm{k})} v_{\mathrm{m}}}{\varepsilon_{\mathrm{sm}}}\left(\mathrm{v}_{\mathrm{g}}\left(\mathrm{x}_{\mathrm{k}}\right)\right. \\
& \left.-\mathrm{v}_{\mathrm{sm}}\left(\mathrm{x}_{\mathrm{k}}\right)\right)
\end{aligned}
$$

onde $P_{\mathrm{g}}\left(\mathrm{x}_{k}\right)$ e $\mathrm{vg}_{\mathrm{g}}\left(\mathrm{x}_{k}\right)$ são os campos pressão média $P_{\mathrm{g}}$ e a velocidade $\mathrm{v}_{\mathrm{g}}$ da fase gasosa da $k^{\text {th }}$ partícula, $\mathcal{V}_{m}$ é o volume da partícula, e $\beta_{m}^{(k)}$ é a o coeficiente de transferência local da quantidade de movimento para a $k^{\text {th }}$ célula.

O $\beta_{m}^{(k)}$ representa o coeficiente de arrasto, com diferentes modelos desenvolvidos, que pode ser escrito por:

$$
\begin{aligned}
\beta_{m}^{(\forall i \in k)}=\beta_{\mathrm{m}}^{(\mathrm{k})}= & \left(\rho_{\mathrm{m}}, \mathrm{D}_{\mathrm{m}}, \mid \mathrm{v}_{\mathrm{sm}}\left(\mathrm{x}_{\mathrm{k}}\right)\right. \\
& \left.-\mathrm{v}_{\mathrm{g}}\left(\mathrm{x}_{\mathrm{k}}\right) \mid, \rho_{\mathrm{g}}, \mu_{\mathrm{g}}\right)
\end{aligned}
$$

onde $\mathrm{x}_{\mathrm{k}}$ é o centro da célula $\mathrm{k}, \mathrm{v}_{\mathrm{g}}\left(X^{(i)}\right)$ é a velocidade média do gás na posição $X^{(i)}$, um valor central de $v_{\mathrm{g}}$ é utilizado. Similarmente, $v_{\text {sm }}$ é a velocidade média para a célula da $\mathrm{m}^{\text {th }}$ fase sólida.

Se considerada uma aproximação de força de arrasto constante para todas as partículas pertencentes a uma célula particular, a transferência de momento da fase gás-sólido $I_{g m}^{(k)}$ é estimada para a célula $k$ como:

$$
\begin{array}{r}
I_{g m}^{(k)}=\varepsilon_{\mathrm{sm}} \nabla \mathrm{P}_{\mathrm{g}}\left(\mathrm{x}_{\mathrm{k}}\right) \\
+\beta_{\mathrm{m}}^{(\mathrm{k})}\left(\mathrm{v}_{\mathrm{g}}\left(\mathrm{x}_{\mathrm{k}}\right)-\mathrm{v}_{\mathrm{sm}}\left(\mathrm{x}_{\mathrm{k}}\right)\right)
\end{array}
$$

\subsection{Modelos de arrasto}

Os modelos de arrasto utilizados nas simulações estão implementados no MFIXDEM e são utilizados em estudos de leitos fluidizados. Tais modelos foram descritos por Ding e Gidaspow (1990); Lathouwers e Bellan (2000), aqui apresentado como Gidaspow Blend; Wen e Yu (1966); Hill, Koch e Ladd (2001), aqui apresentado apenas como Hill; e Beetstra, van der Hoef e Kuipers (2007), ou apenas BVK.

\section{RESULTADOS E DISCUSSÕES}

Os resultados foram obtidos com auxílio da ferramenta POST do MFIX, cuja função foi calcular a média dos valores da pressão no leito em intervalos de tempo regulares. A partir dos resultados obtidos foram elaboradas as curvas de perda de carga pela velocidade de entrada do ar. Os pontos das linhas tracejadas representam os dados experimentais extraídos de Soponronnarit et al. (2001). Já os pontos das linhas contínuas representam as médias dos valores numéricos obtidos por meio das simulações computacionais. Os valores médios foram obtidos considerando todos os passos de tempo simulados durante os $3 \mathrm{~s}$ para cada velocidade de entrada do ar, conforme mostrado na Figura 2, Figura 3 e Figura 4.

$\mathrm{Na}$ Figura 2 são apresentados resultados de perda de carga para diferentes coeficientes de arrasto para a altura de leito estático de 
0,080 m. Pode-se verificar que os modelos BVK e Koch-Hill-Ladd apresentam um comportamento fora do esperado, apresentando mínima fluidização a uma velocidade de $2,3 \mathrm{~m} / \mathrm{s}$, sendo que para os modelos de Gidaspow, Gidaspow-Blend e Syamlal O'Brien a mínima fluidização ocorreu a uma velocidade de $1,9 \mathrm{~m} / \mathrm{s}$.

A mínima fluidização ocorreu a velocidades superiores também para as alturas de leito estático de $0,115 \mathrm{~m}$ e $0,150 \mathrm{~m}$ quando considerado os modelos de coeficientes de arrasto BVK e Koch-Hill-Ladd, isto pode ser observado na Figura 3 e na Figura 4. Nota-se que a velocidade de mínima fluidização para estes casos se mantém em $2,3 \mathrm{~m} / \mathrm{s}$ enquanto que para os demais coeficientes de arrasto a fluidização ocorre entre $1,9 \mathrm{~m} / \mathrm{s}$ e $2,0 \mathrm{~m} / \mathrm{s}$.

Figura 2 - Diagrama da Perda de Carga versus Velocidade de Entrada do Ar para $h_{\text {leito }}=0,080 \mathrm{~m}$.

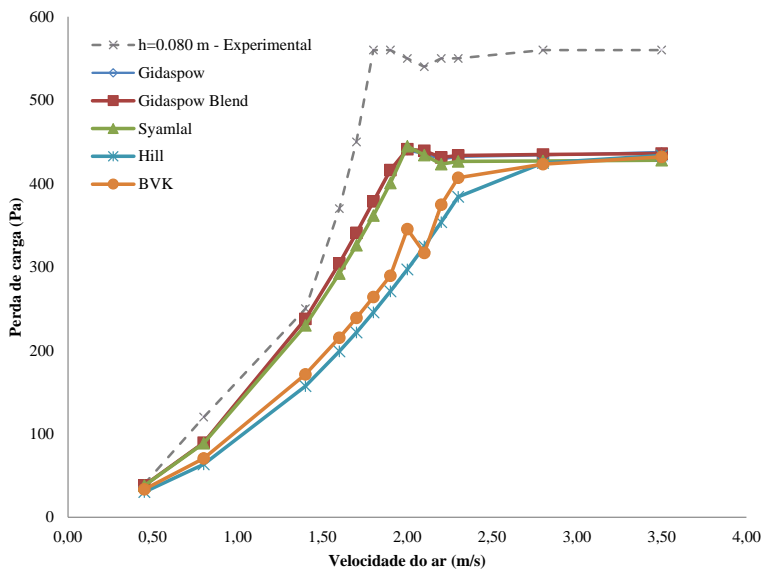

Fonte: Os autores (2015)

Verificou-se nos resultados obtidos que existe um aumento da perda de carga com o aumento da altura do leito estático de partículas. Isto é esperado, já que para uma aumentar a altura de leito estático é necessário um maior número de partículas, o que implica em um maior peso do conjunto. Desta forma faz-se necessária uma maior perda de carga para equilibrar o peso aparente do leito.
Figura 3 - Diagrama da Perda de Carga versus Velocidade de Entrada do Ar para $h_{\text {leito }}=0,115 \mathrm{~m}$.

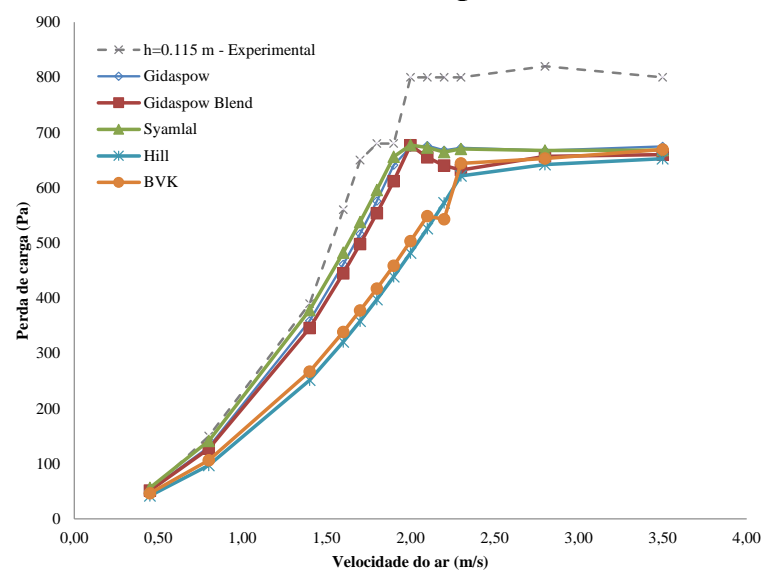

Fonte: Os autores (2015)

No entanto, quando consideradas as velocidades de mínima fluidização não se evidencia a influência da altura do leito estático. Isto se deve ao fato de que o diâmetro médio de partículas utilizado foi o mesmo para todos os grupos de simulação.

Figura 4 - Diagrama da Perda de Carga versus Velocidade de Entrada do Ar para $h_{\text {leito }}=0,150 \mathrm{~m}$.

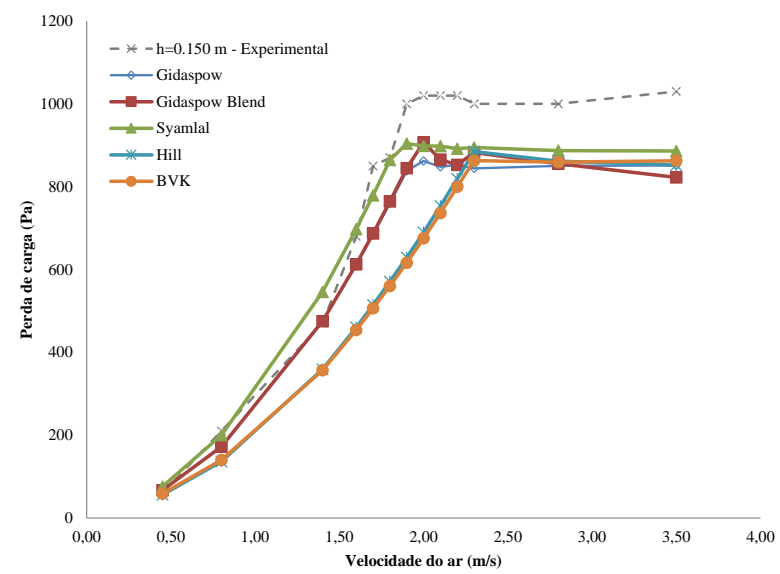

Fonte: Os autores (2015)

Na Figura 5 são mostrados os valores de porosidade do leito pela velocidade de entrada do ar para a altura de leito estático de $0,080 \mathrm{~m}$, considerando-se os coeficientes de arrasto de Syamlal O'Brien, BVK e Koch-Hill-Ladd. 
Figura 5 - Diagrama da Porosidade versus Velocidade de Entrada do Ar para o leito preenchido com partículas até $h_{\text {leito }}=0,080 \mathrm{~m}$.

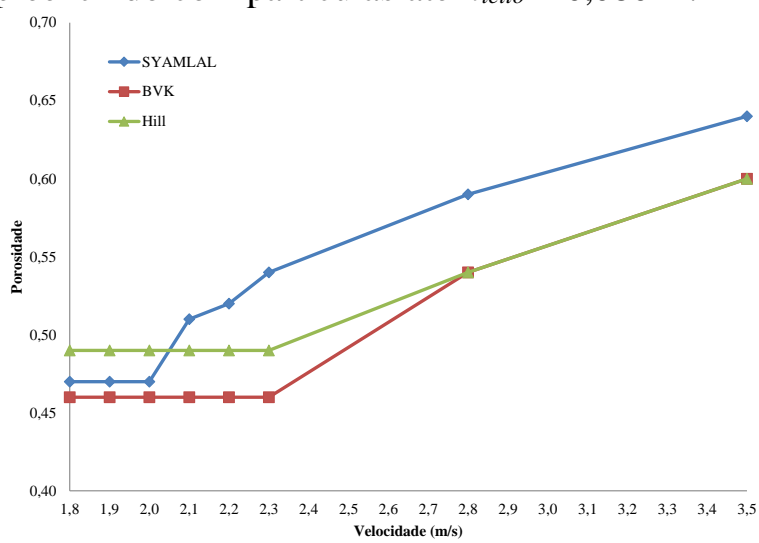

Fonte: Os autores (2015)

Verifica-se que para as condições de leito fixo, ou seja, abaixo da velocidade de mínima fluidização de $1,9 \mathrm{~m} / \mathrm{s}$ para Syamlal O'Brien e de $2,3 \mathrm{~m} / \mathrm{s}$ para BVK e Koch-HillLadd, que os valores de porosidade permanecem constantes e equivalentes a 0,49 , 0,46 e 0,47 respectivamente. Verifica-se também um comportamento adequado do regime de fluidização, uma vez que, as porosidades aumentaram quando as velocidades foram superiores as de mínima fluidização. Também é possível dizer que a porosidade na mínima fluidização obtida pelos coeficientes de arrasto analisados apresentam valores próximos da porosidade experimental, obtida através do modelo de Ergun, com valor de 0,48 .

\section{CONCLUSÕES}

O método DEM acoplado ao CFD apresentou bons resultados para predizer a velocidade de mínima fluidização dos casos estudados. Os valores numéricos resultantes dos modelos de Gidaspow, Gidaspow-Blend e de Syamlal O'Brien, pouco diferem dos valores experimentais. Já os modelos de arrasto de BVK e Koch-Hill-Ladd, apresentaram velocidades de mínima fluidização maiores que os resultados experimentais do caso estudado.

Com relação aos valores de perda de carga, as diferenças entre os valores numéricos e experimentais para são mais significativas, apesar do comportamento das curvas ser parecido. Isto pode estar atrelado ao fato de que muitas das variáveis foram baseadas em dados da literatura e não do próprio material analisado experimentalmente. Além disso, a diferença nos resultados pode ser devido à recirculação existente no leito, que altera a pressão.

\section{NOMENCLATURA}

\section{Notação}

$D$ diâmetro da partícula, $\mathrm{m}$

$\overline{\bar{D}}_{g}$ taxa de deformação para a fase fluida, $\mathrm{s}^{-1}$

$D_{m} \quad$ diâmetro da partícula

$F$ força, $\mathrm{N}$

$F_{C}$ força de contato, $\mathrm{N}$

$F_{D}$ força de arrasto, $\mathrm{N}$

$F_{T}$ força total, N

$g$ aceleração da gravidade, $\mathrm{m} / \mathrm{s}^{2}$

$h$ altura, $\mathrm{m}$

I momento de inércia, $\mathrm{kg} / \mathrm{m}^{2}$

$I_{g m}$ transferência de momento da fase fluida/sólida, $\mathrm{N} / \mathrm{m}^{3}$

$N_{m} \quad$ número de partículas esféricas

$P$ pressão, $\mathrm{Pa}$

$P_{g} \quad$ pressão para a fase gasosa, $\mathrm{Pa}$

$\overline{\bar{S}}_{g} \quad$ tensor tensão da fase gasosa, $\mathrm{Pa}$

$T$ torque, $\mathrm{N} / \mathrm{m}$

$u_{m f} \quad$ velocidade de mínima fluidização, $\mathrm{m} / \mathrm{s}$

$v \quad$ velocidade, $\mathrm{m} / \mathrm{s}$

$\vec{v}_{g} \quad$ vetor velocidade para a fase gasosa, $\mathrm{m} / \mathrm{s}$

$v_{\text {sm }} \quad$ velocidade para a fase sólida, $\mathrm{m} / \mathrm{s}$

$V \quad$ velocidade linear, $\mathrm{m} / \mathrm{s}$

$\mathcal{V}_{m} \quad$ volume da partícula, $\mathrm{m}^{3}$

$X$ posição, $\mathrm{m}$ 
Letras gregas

$\beta_{m} \quad$ coeficiente de transferência local da quantidade de movimento

$\varepsilon_{g} \quad$ fração volumétrica da fase gasosa

$\rho \quad$ densidade, $\mathrm{kg} / \mathrm{m}^{3}$

$\rho_{g} \quad$ densidade da fase gasosa, $\mathrm{kg} / \mathrm{m}^{3}$

$\rho_{\text {sm }} \quad$ densidade da $\mathrm{m}^{\text {th }}$ fase sólida, $\mathrm{kg} / \mathrm{m}^{3}$

$\overline{\bar{\tau}}_{g} \quad$ tensor tensão no regime viscoso, $\mathrm{Pa}$

$\omega \quad$ velocidade angular, $\mathrm{s}^{-1}$

\section{REFERÊNCIAS}

ANDERSON, T.B., JACKSON, R. A Fluid Mechanical Description of Fluidized Beds Equations of Motion, Industrial e Engineering Chemistry Fundamentals, Vol. 6 (4), p. 527-539, 1967.

ASEGEHEGN, T. W., KRAUTZ, H. J. SCHREIBER, M. Numerical Study of a GásSólid Fluidized Beds Hydrodynamics: Influence of Immersed Horizontal Tubes and Data Analysis. International Journal of Chemical Reactor Engineering, Vol. 9 (1), p. 1-39, 2011.

BEETSTRA, R, VAN DER HOEF, M. F., KUIPERS J. A. M. Numerical study of segregation using a new drag force correlation for polydisperse systems derived from latticeBoltzmann simulations, Chemical Engineering Science, Vol. 62 (1), 246-255, 2007.

BOAC, J.M., CASADA, M.E., MAGHIRANG, R.G., HARNER, J.P. Material and Interaction Properties of Selected Grains and Oilseeds for Modeling Discrete Particles. ASABE Annual International Meeting, Paper Number 09-7166, 2009.
DUARTE, C.R., OLAZAR, M., MURATA, V.V., BARROZO, M.A.S. Numerical simulation and experimental study of fluidparticle flows in a spouted bed. Powder Technology, Vol. 188, p. 195-205, 2009.

DING, J., GIDASPOW, D. A bubbling fluidization model using kinetic theory of granular flow, AIChE Journal, Vol. 36 (4), 523-538, 1990.

GELDART, D. Expansion of Gas Fluidized Beds. Industrial e Engineering Chemistry Research, Vol. 43 (18), p. 5802-5809, 2004.

GIDASPOW, D. Multiphase Flow and Fluidization: Continuum and Kinetic Theory Descriptions. Academic Press, Boston, 1994.

GIDASPOW, D., BEZBURUAH, R., DING, J. Hydrodynamics of circulating fluidized beds, kinetic theory approach in fluidization, Proceedings of the 7th Engineering Foundation Conference on Fluidization, p. 75-82, 1992.

HILL R. J., KOCH D. L., LADD J. C. The first effects of fluid inertia on flows in ordered and random arrays of spheres, Journal of Fluid Mechanics, 448, 213-241, 2001.

HULME, I., CLAVELLE, E., VAN DER LEE, L., KANTZAS, A. CFD Modeling and Validation of Bubble Properties for a Bubbling Fluidized Bed. Industrial e Engineering Chemistry Research, Vol. 44 (12), p. 42544266, 2005.

KUNII, D., LEVENSPIEL, O. Fluidization Engineering. John Wiley \& Sons: New York, p. 491, 1991.

LATHOUWERS, D., BELLAN, J. Modeling of dense gas-solid reactive mixtures applied to biomass pyrolysis in a fluidized bed, 
Proceedings of the 2000 U.S. DOE Hydrogen Program Review NREL/CP-57028890, 141-203, 2000.

LAW, C. L., MUJUMDAR, A. S. Fluid Bed Dryers. In Handbook of Industrial Drying. Taylor \& Francis. Cap. 8, p 174-199, 2006.

LOFSTRAND, H., ALMSTED, A. E.,ANDERSON, S. Dimensionless Expansion Model for Bubbling Fluidized Beds with and without Internal Heat Exchanger Tubes. Chemical Engineering Science, Vol. 50 (2), p. 245-253, 1995.

SOPONRONNARIT, S., SWASDISEVI, T., WETCHACAMA, S., WUTIWIWATCHAI, W. Fluidised bed drying of soybeans. Journal of Stored Products Research, Vol. 37, p. 133151, 2001.

SYAMLAL, M., O'BRIEN, T.J. Computer simulation of bubbles in a fluidized bed. AIChE Symp. Ser., Vol. 85, p. 22-31, 1989.

TSUJI, Y., KAWAGUCHI, T. AND TANAKA, T. Discrete particle simulation of two-dimensional fluidized bed. Powder Technology, Vol. 77, p. 79-87, 1993.

TAGHIPOUR, F., ELLIS, N., WONG, C. Experimental and computational study of gassolid fluidized bed hydrodynamics, Chem. Eng. Sci., Vol. 60, p. 6857-6867, 2005.

WEN, C.Y., YU, Y.H. Mechanics of fluidization, Chem. Eng. Prog. Symp. Ser., Vol. 62, p. 100-111, 1966.

\section{AGRADECIMENTOS}

Os autores agradecem a CAPES e a Fundação Araucária pelo suporte financeiro e o Centro Nacional de Supercomputação (CESUP) da Universidade Federal do Rio Grande do Sul pela disponibilidade de recursos e tecnologia para o desenvolvimento desta pesquisa. 